\title{
DEFINITIONS AND CONVENTIONS
}

SEVERAL CONVENTIONS ARE EMPLOYED IN THE BOOK. "Egyptian-style" or "Egyptian" refers to hard stone and other statues created by native craftsmen, even those that display strong Greek influence. "Greek-style" or "Greek" describes statues made by Greeks, typically in marble or bronze. Like terminology denotes whether a temple displays predominantly Greek or Egyptian features. When an Egyptian sculpture has "Greek hair" (Fig. 105), it means that the hair is patterned after Greek-style works (Fig. 260), even though it may be rendered in a stylized Egyptian manner. The same is true for "Roman hair."

For the hieroglyphic and demotic texts, I use the European system of transliteration, ${ }^{1}$ regardless of whether it was used by authors in the citations for these texts. For the Greek, I provide a transliteration unless an anglicized form is better known, such as "Ptolemy." For common Egyptian terms, I use established transliterations, such as the shendyt-garment or heqa-scepter. Egyptological convention is inconsistent in using a Greek, Roman, or Arabic name for sites. ${ }^{2}$ Although I prefer the Greek or Roman designation, I often employ the Arabic one because it is more commonly cited. For example, I use the Arabic Medinet Madi rather than the Greek Narmouthis, because Medinet Madi typically appears in major publications on the site.

Table D.1 gives the conventions employed for the purpose of classifying sculptures into broad categories of size. In addition, some special terminology describes the depiction of the eyes and eyebrows:

- Eyebrows in relief = indicated in relief edged by incision, generally in a hieroglyphic, symmetrical manner (Fig. 20).

- Natural eyebrows $=$ rendered as raised contours that are not edged by incision (Fig. 52).

- Single-arc versus semicircular eyebrows = executed as a continuous, single curve versus in two separate semicircles (Fig. 199). 
TABLE D. 1 HEIGHT CONVENTIONS

(in centimeters)

\begin{tabular}{lcc}
\hline & $\begin{array}{l}\text { Height of Head } \\
(=\text { HeadH })\end{array}$ & $\begin{array}{c}\text { Height of Face } \\
(=\text { FaceH })^{\mathrm{a}}\end{array}$ \\
\hline Well under life-size & Up to 9.1 & Up to 6.2 \\
Under life-size & $9.2-21.1$ & $6.3-13 \cdot 3$ \\
Life-size & $21.2-27.3$ & $13 \cdot 4-18.6$ \\
Over life-size & $27.4-40.0$ & $18.7-26.0$ \\
Colossal & 40.1 and up & 26.1 and up \\
\hline
\end{tabular}

${ }^{a}$ Forehead hair, if any, is excluded from the height of face measurements.

- Rimmed eyes = upper lid of the eye is rendered plastically, with both of its edges indicated by incision. Only the upper edge of the lower lid is marked with incision (Fig. 138).

- Fully rimmed eyes = both upper and lower lids are rendered plastically, with both edges of each lid indicated in relief edged by incision (Fig. 158).

Catalogued sculptures are arranged into Groups A-J and designated by a number, such as $\mathrm{A} 3$ or $\mathrm{D} 8$. The sequence of groups, which is mostly chronological, is explained at the beginning of Chapter 7 and in Table 7.1.
Every effort has been made over the last several years to obtain photographs, but not all statues are illustrated. Catalogued sculptures generally are referenced by their catalogue numbers (such as A3), whereas other objects are labeled by their figure numbers (as in Fig. 240). Left and right sides refer to a statue's own left and right. Exceptionally, the viewer's left or right is used in reference to inscriptions, reliefs, or temple entrances.

Question marks (?) next to a sculptural reference, transliteration, date, or another word indicate that the description, reading, dating, or interpretation is uncertain. Thus, in citing a list of sculptures with double crowns, the number of a catalogued sculpture is followed by a question mark (as in “В7?”), if it is not certain that the crown was originally present. I use the traditional B.C. and A.D dating designations. When neither is specified, B.C. should be understood. Despite attempts at standardizing to these conventions, inconsistencies undoubtedly persist in the text.

\section{NOTES}

1. James P. Allen, Middle Egyptian: An Introduction to the Language and Culture of Hieroglyphs (Cambridge, 2000), 14-15.

2. See comments of Di. Arnold 1999, 4. 